\title{
Photoinduced Bimolecular Electron Transfer Investigated by Femtosecond Time-Resolved Infrared Spectroscopy
}

\author{
Omar F. Mohammed, ${ }^{\dagger,}$ Natalie Banerji, ${ }^{\dagger}$ Bernhard Lang, ${ }^{\dagger}$ Erik T. J. Nibbering,,$* \hbar$ and \\ Eric Vauthey*, $\dagger$ \\ Department of Physical Chemistry, University of Geneva, 30 quai Ernest-Ansermet, CH-1211 Geneva 4, \\ Switzerland, Max Born Institut für Nichtlineare Optik und Kurzzeitspektroskopie, Max-Born-Strasse 2 A, \\ D-12489 Berlin, Germany
}

Received: September 18, 2006; In Final Form: October 30, 2006

\begin{abstract}
Ultrafast infrared transient absorption spectroscopy is used to study the photoinduced bimolecular electron transfer reaction between perylene in the first singlet excited state and 1,4-dicyanobenzene in acetonitrile and dichloromethane. Following vibrational marker modes on both donor and acceptor sides in real time provides direct insight into the structural dynamics during the reaction. A band narrowing on a time scale of a few tens of picoseconds observed on the antisymmetric $\mathrm{CN}$ stretching vibration of the dicyanobenzene radical anion indicates that a substantial part of the excess energy is channeled into vibrational modes of the product, despite the fact that the reaction is weakly exergonic. An additional narrowing of the same band on a time scale of several hundreds of picoseconds observed in acetonitrile only is interpreted as a signature of the dissociation of the geminate ion pairs into free ions.
\end{abstract}

\section{Introduction}

Since the pioneering work of Weller and co-workers, ${ }^{1,2}$ bimolecular photoinduced electron transfer (ET) reactions have been intensively investigated. ${ }^{3-5}$ These processes are the simplest bimolecular reactions and have thus been the object of many theoretical treatments. ${ }^{6-8}$ Furthermore, they play an important role in many areas of chemistry and biology and are involved in several practical applications such as solar energy conversion, ${ }^{9}$ and photopolymerization. ${ }^{10}$ Despite the numerous studies carried out on bimolecular ET, important questions still remain unanswered, ${ }^{5}$ such as the nature of the primary ET quenching product and the various steps leading to the formation of free solvated ions in polar solvents. A major experimental problem is that ion pairs and free ions cannot be differentiated from their electronic absorption spectra in the visible region. Interionic charge recombination transitions ${ }^{11}$ have never been reported for such ion pairs. This might be due to an intrapair coupling that is insufficient to result in a significant oscillator strength and/or to the presence of these transitions in the nearinfrared, as recently observed with bianthryl in acetonitrile. ${ }^{12}$ Weller and co-workers have also shown that the magnetic field effect could give some insight into this problem. ${ }^{13}$ However, the time resolution of their experiments was not sufficient to directly monitor the dissociation of ion pairs into free ions.

Very recently, we have shown that the dynamics of the ground state recovery of a radical ion after optical excitation is not the same when the ion is paired or free in solution and that this difference can be used to monitor the dissociation of the pair into free ions. ${ }^{14}$ However, the pump-pump-probe technique used for such measurements suffers from substantial experimental difficulties and constraints. A more promising approach

\footnotetext{
* Corresponding authors. E-mail: nibberin@mbi-berlin.de (E.T.J.N.), eric.vauthey@chiphy.unige.ch (E.V.).

$\dagger$ University of Geneva.

$\doteqdot$ Max Born Intitut für Nichtlineare Optik und Kurzzeitspektroskopie.
}

to obtain direct structural information is time-resolved vibrational spectroscopy. Over the past few years, there have been several reports on the application of time-resolved infrared (IR) and resonance Raman spectroscopies for investigating charge transfer processes. ${ }^{15}$ Most of these studies were related to intramolecular processes in transition metal complexes, ${ }^{16,17}$ in organic molecules, ${ }^{18,19}$ or in covalently linked donor-acceptor assemblies, ${ }^{20,21}$ to ET processes in biological systems, ${ }^{22,23}$ or to electron injection in dye/semiconductor systems. ${ }^{24-26} \mathrm{Few}$ studies on photoinduced intermolecular ET reactions have been published so far. Some of them are related to ET between an excited chromophore and the solvent. ${ }^{27-29}$ In most studies on bimolecular reactions, vibrational spectroscopy was essentially used to differentiate the ET product, namely the radical ions, from the products of other processes, such as triplet energy and proton transfer. ${ }^{30-32}$ Several years ago, a nanosecond resonance Raman investigation was reported, where ET between anthraquinone in the lowest triplet state and trimethoxybenzene in solvents of various polarity was studied. ${ }^{33}$ Apart from one exception, all the observed ion bands exhibited neither a solvent nor a time dependence in their shape or frequency. Only in one case was the frequency of a band assigned to the $\mathrm{C}-\mathrm{C}$ stretching of the trimethoxybenzene radical cation found to be solvent dependent. Moreover, in a solvent of medium polarity, a temporal dependence on the $100 \mathrm{~ns}$ time scale was observed and assigned to the dissociation of the ion pair into free ions. ${ }^{34}$ The time resolution of this experiment was, however, not sufficient to observe this process in acetonitrile, where dissociation typically occurs on a sub-nanosecond time scale. ${ }^{35,36}$ Furthermore, ET quenching of molecules in the singlet excited state has not been investigated with time-resolved Raman spectroscopy, because the weak signals are in most cases hidden by a strong fluorescence background. Recently, time-resolved resonance Raman measurements of highly fluorescent compounds using a Kerr shutter have been demonstrated. ${ }^{37}$ However, this approach makes the experiment rather complex and limits 


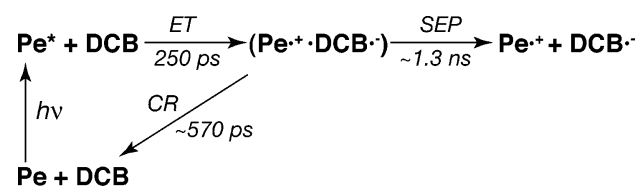

Figure 1. Reaction scheme for the Pe/DCB pair in $\mathrm{ACN}$ and relevant time constants taken from ref 14 . The $250 \mathrm{ps}$ value corresponds to the average time constant of Pe fluorescence quenching by $0.16 \mathrm{M} \mathrm{DCB}$, the dynamics being nonexponential because of the transient effect. The two remaining time constants have been deduced from all-visible pump-pump-probe experiments.

the time resolution to about 2 ps. For these reasons, timeresolved IR spectroscopy seems to be better suited to investigate such ultrafast ET quenching processes.

Here, we report on an investigation of the bimolecular ET quenching reaction of perylene $(\mathrm{Pe})$ and 1,4-dicyanobenzene (DCB) in acetonitrile and dichloromethane using time-resolved IR spectroscopy. This donor-acceptor pair has already been studied in acetonitrile using the above-mentioned all-visible pump-pump-probe technique, ${ }^{14}$ and the results are summarized in Figure 1. By recording transient IR absorption spectra of the radical ions generated upon photoinduced ET and analyzing height and width of the corresponding bands, we can directly follow the generation of both products under identical conditions, which is not possible with Pe and DCB in an all-visible experiment. A careful shape analysis of the $\mathrm{CN}$ stretching band of the DCB radical anion allows the influence of the counterion proximity on the band shape to be studied, resulting in a direct insight into the dissociation of the geminate ion pair into free ions.

\section{Experiment}

Femtosecond $400 \mathrm{~nm}$ pump pulses were generated by frequency doubling the output of a home-built Ti:sapphire regenerative laser system operating at $800 \mathrm{~nm} .{ }^{38}$ The energy per pulse at the sample was about $1-2 \mu \mathrm{J}$, with a pulse duration of $55 \mathrm{fs}$ and a spot size of $500 \mu \mathrm{m}$ diameter. Mid-IR pulses were generated with typical energies of $0.7-1 \mu \mathrm{J}$ per pulse using double-pass collinear optical parametric amplification based on a BBO nonlinear crystal followed by difference frequency mixing of signal and idler. ${ }^{39}$ The mid-IR probe and reference pulses, split off by a ZnSe wedge, were spectrally dispersed in a grating spectrometer and recorded simultaneously by singleshot detection using a liquid nitrogen cooled double array $(2 \times$ 32 pixels) of MCT ( $\mathrm{HgCdTe})$ detectors with $2-7 \mathrm{~cm}^{-1}$ spectral resolution. Synchronous chopping of the pump pulses was used to eliminate long-term drift effects. The whole pump-probe setup was purged with nitrogen to avoid spectral and temporal reshaping of the mid-IR pulses by the absorption of water vapor and $\mathrm{CO}_{2}$ in air.

The time resolution was determined to be $150 \mathrm{fs}$ based on cross-correlation measurements between $400 \mathrm{~nm}$ pump and IR probe pulses and on estimations of group velocity mismatch between both pulses. On several occasions, the $400 \mathrm{~nm}$ pump pulses were stretched to 300 fs duration using a water cell to suppress multiphoton contributions. In particular, at the shortest possible pump pulse duration, the transient $\mathrm{CN}$ stretching band is superimposed to a broad and time-dependent background, which we tentatively ascribe to a direct two-photon ionization of $\mathrm{Pe},{ }^{40}$ with subsequent solvation of the free electron or capturing of the electron by a nearby DCB molecule.

Perylene (Pe, Aldrich $>99.5 \%$ ) was used as received and 1,4dicyanobenzene (terephthalonitrile, DCB) was sublimed before use. The perdeuterated solvents acetonitrile $\left(\mathrm{ACN}-d_{3}\right)$, dichlo-

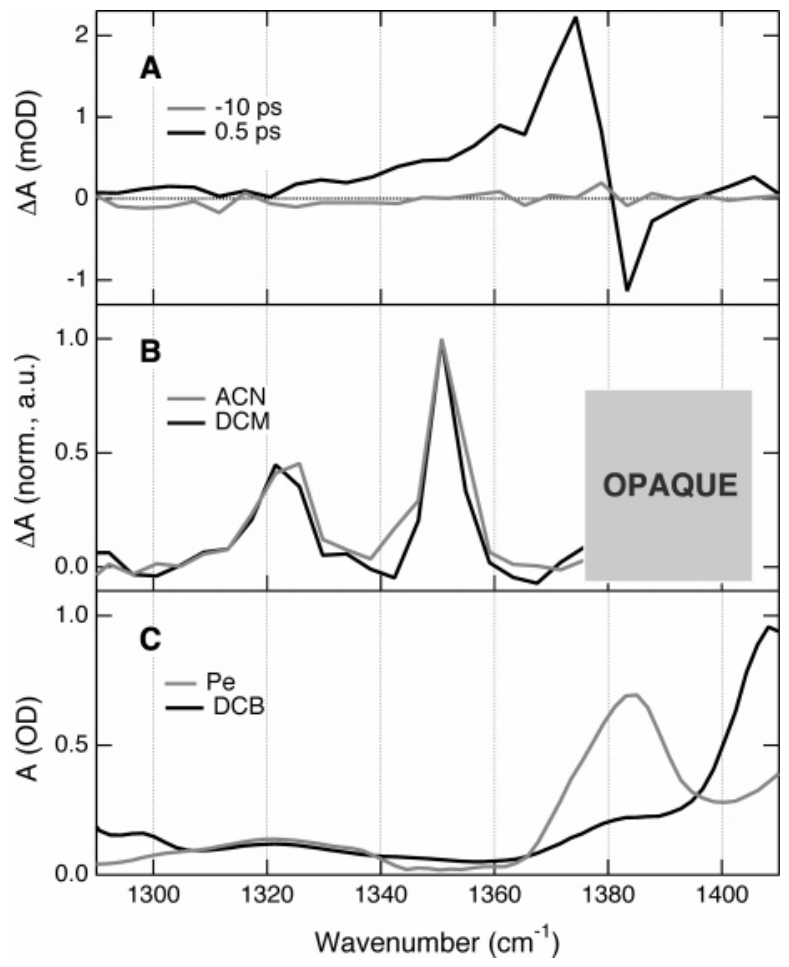

Figure 2. (A) Transient IR spectrum measured with Pe in DMSO- $d_{6}$ $0.5 \mathrm{ps}$ after $400 \mathrm{~nm}$ excitation. A spectrum at negative time delay is also shown for comparison. (B) Transient IR spectra measured with perylene and $0.16 \mathrm{M} \mathrm{DCB}$ in $\mathrm{ACN}-d_{3}$ and $\mathrm{DCM}-d_{2} 700$ ps after $400 \mathrm{~nm}$ excitation. (C) Steady state IR spectra of Pe and DCB in DMSO- $d_{6}$.

romethane (DCM- $\left.d_{2}\right)$ and dimethyl sulfoxide (DMSO- $d_{6}$ ) were obtained from Deutero $\mathrm{GmbH}$ (99.8\% deuteration grade), and the other solvents acetonitrile (ACN) and dichloromethane (DCM) were purchased from Merck (p.a.). All solvents were used without further purification. The sample solutions were circulated through a flow cell consisting of $1 \mathrm{~mm}$ thick $\mathrm{BaF}_{2}$ windows separated by a $200 \mu \mathrm{m}$ thick Teflon spacer, guaranteeing that for every laser shot a new sample volume was excited. All experiments were performed at room temperature $(23 \pm 2$ $\left.{ }^{\circ} \mathrm{C}\right)$.

\section{Results and Discussion}

Immediately upon excitation of Pe with a $400 \mathrm{~nm}$ pump pulse, bands due to vibrational modes of $\mathrm{Pe}$ in both the electronic ground state, $\mathrm{Pe}\left(\mathrm{S}_{0}\right)$, and the lowest singlet excited state, $\mathrm{Pe}$ $\left(\mathrm{S}_{1}\right)$, appear as bleach and absorption signals, respectively. Figure $2 \mathrm{~A}$ shows a transient absorption-bleach pair measured $0.5 \mathrm{ps}$ after impulsive excitation of Pe at $400 \mathrm{~nm}$ in DMSO- $d_{6}$. Bands with a good signal-to-noise ratio in this frequency region required a high Pe concentration ( $\geq 5 \mathrm{mM}$ ), which can only be realized in DCM and DMSO. In the less viscous DCM, the Pe excited lifetime is substantially reduced because of the occurrence of self-quenching, which is known to result to the formation of both radical cation and anion of Pe. ${ }^{41}$ The more viscous DMSO was thus chosen here to minimize this effect.

Figure 2B shows the same spectral region measured with 0.16 M DCB as electron acceptor, $700 \mathrm{ps}$ after $400 \mathrm{~nm}$ excitation in both deuterated ACN and DCM. The measurements with DCB were not performed in DMSO, because its high viscosity makes diffusional ET quenching too slow for the time window of the experiment. Moreover, self-quenching of Pe in DCM is negligible at such high DCB concentration. The peaks at 1315 and $1346 \mathrm{~cm}^{-1}$ can be assigned to the Pe radical cation, the 
frequencies being in good agreement with those of the two most intense IR bands of $\mathrm{Pe}^{\cdot+}$ reported in the literature. ${ }^{42}$ The lowfrequency band exhibits a slight dependence on the solvent, and the other is not or is barely influenced by the solvent. The $\mathrm{S}_{0}$ bleaching and $S_{1}$ absorption of Pe cannot be measured in the presence of DCB because the solution becomes opaque in this frequency region due to a nearby absorption band of DCB (see Figure 2C).

Figure $3 \mathrm{~A}$ depicts the band originating from the antisymmetric $\mathrm{CN}$ stretching mode of $\mathrm{DCB}^{\bullet-}$. A $4 \mathrm{~cm}^{-1}$ shift and a broadening by almost a factor of 2 are observed when going from ACN to DCM. This band is downshifted by about $135 \mathrm{~cm}^{-1}$ with respect to the $\mathrm{CN}$ stretching band of the neutral $\mathrm{DCB}$, pointing to a substantial increase of electron density in the $\mathrm{CN}$ groups upon electron attachment to DCB. The solvent dependence of the frequency of this mode indicates that the distribution of the excess electric charge in $\mathrm{DCB}^{--}$depends on the environment of the anion. This IR band is thus a promising candidate for probing the presence or absence of the counterion in the proximity of $\mathrm{DCB}^{\bullet-}$ after ET. Solvent-induced vibrational frequency shifts have also been observed before in hydrogenbonding dynamics, ${ }^{43-45}$ and in metallocarbonyl compounds. ${ }^{46}$

An analysis of the band intensity as a function of time delay allows the rate constants of product formation to be extracted. Figure $3 \mathrm{~B}$ shows the measured time-profiles of the intensity of the $\mathrm{DCB}^{--}$band at $2103 \mathrm{~cm}^{-1}$ and of the $\mathrm{Pe}^{\bullet+}$ band at 1346 $\mathrm{cm}^{-1}$. Both kinetics are identical, which clearly indicates that the formation of the ionic product does not involve a third reactant, such as a solvent molecule, or an electronic excited state of one of the ions. This is not surprising for this donoracceptor pair. For comparison, the time evolution of the absorbance at $532 \mathrm{~nm}$, associated with the $\mathrm{D}_{0}-\mathrm{D}_{5}$ electronic transition of $\mathrm{Pe}^{\bullet+}$ and measured with the same DCB concentration, ${ }^{14}$ is also shown in Figure $3 \mathrm{C}$ together with that of the $\mathrm{CN}$ band of $\mathrm{DCB}^{\bullet-}$. Although the kinetics shown here do not contain new information with respect to what is already known for this donor-acceptor (DA) pair, they demonstrate the potential of time-resolved mid-IR spectroscopy. $\mathrm{DCB}^{\bullet-}$ does not exhibit electronic absorption bands in the visible spectral region, ${ }^{47}$ and thus the information provided by the comparison of the kinetics of both $\mathrm{Pe}^{\bullet+}$ and $\mathrm{DCB}^{\bullet-}$ cannot be obtained directly from conventional all-visible transient absorption measurements. Even with DA pairs where both products exhibit all electronic transitions in the visible, a detailed analysis of the bands is often difficult to perform due to the large spectral broadening, which leads to strongly overlapping bands. The comparison of the results obtained by probing electronic and vibrational transitions of $\mathrm{Pe}\left(\mathrm{S}_{1}\right)$ and $\mathrm{Pe}^{\bullet+}$ provides a check for the consistency of the measured data. Finally, Figure 3D shows a comparison of the kinetics of the $\mathrm{CN}$ stretching vibration measured in $\mathrm{ACN}$ and DCM. The slower kinetics in DCM is consistent with a decrease of the ET driving force upon going from a polar to a weakly polar solvent. Indeed, the driving force, calculated with the Weller equation, ${ }^{48}$ amounts to -0.26 and $-0.1 \mathrm{eV}$ in $\mathrm{ACN}$ and DCM, respectively. With $0.16 \mathrm{M}$ DCB, ET quenching is complete about half a nanosecond after photoexcitation.

Further information on the reaction dynamics can be obtained from an analysis of the shape of the different product bands observed as a function of time delay. Figures 4 and 5 show the results of such an analysis for the $\mathrm{CN}$ stretching vibration of $\mathrm{DCB}^{--}$in both solvents. In Figure 4, the top panels depict the measured data and the bottom panels the same data normalized to the peak maximum, and Figure 5 shows the temporal evolution of the full width at half-maximum (fwhm) and of the
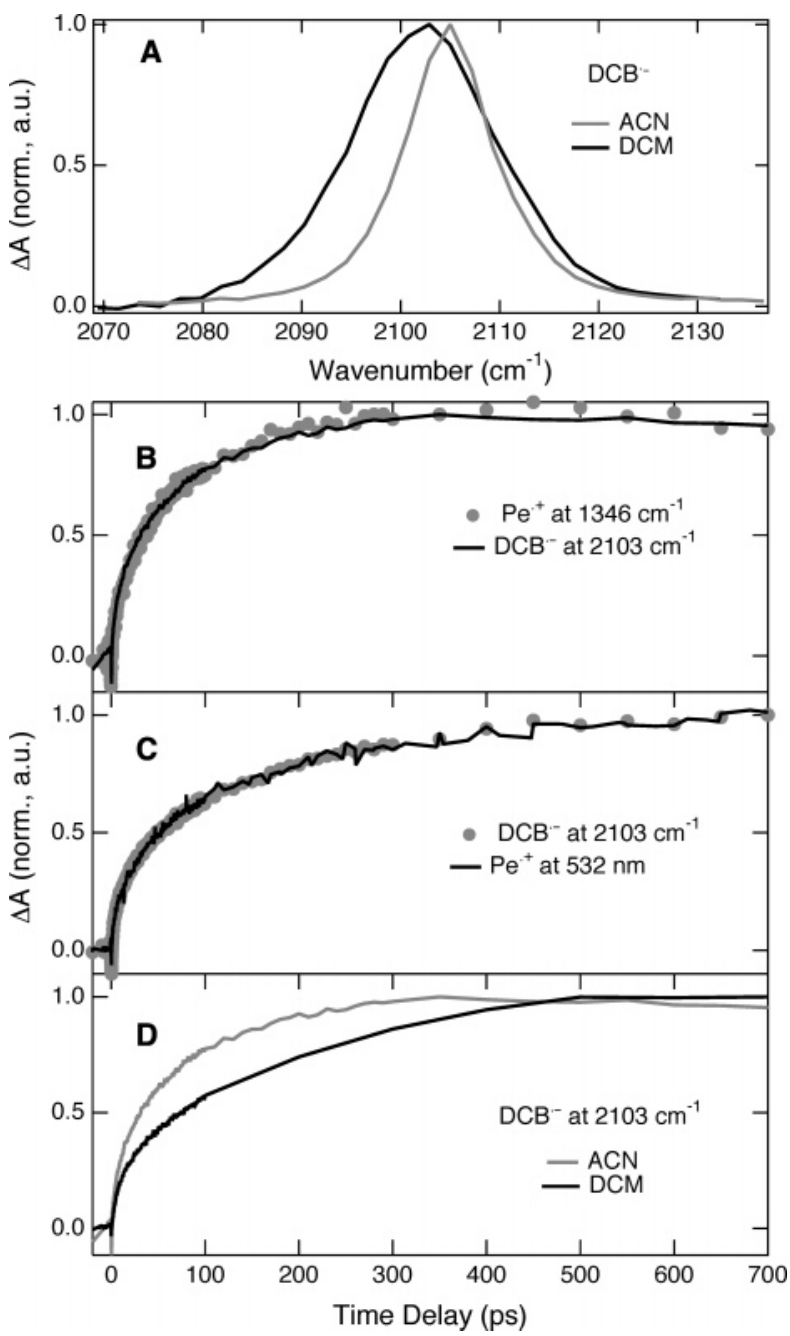

Figure 3. (A) IR absorption band associated with the antisymmetric $\mathrm{CN}$ stretching mode of $\mathrm{DCB}^{--}$measured 700 ps after excitation of Pe and $0.16 \mathrm{M}$ DCB in ACN and DCM. (B) Time profiles of the intensity of the $1346 \mathrm{~cm}^{-1}$ band of $\mathrm{Pe}^{\bullet+}$ and of the $2103 \mathrm{~cm}^{-1}$ band of $\mathrm{DCB}^{\bullet-}$ measured upon excitation of Pe and 0.16 M DCB in ACN. (C) Time profiles of the $2103 \mathrm{~cm}^{-1}$ band of $\mathrm{DCB}^{\cdot-}$ and of the $\mathrm{D}_{0}-\mathrm{D}_{5}$ absorption band of $\mathrm{Pe}^{\bullet+}$ at $532 \mathrm{~nm}$ (taken from ref 14) measured upon excitation of Pe and 0.1 M DCB in ACN. (D) Time profiles of the intensity of the $\mathrm{CN}$ stretching band of $\mathrm{DCB}^{--}$measured upon excitation of $\mathrm{Pe}$ and $0.16 \mathrm{M}$ DCB in $\mathrm{ACN}$ and $\mathrm{DCM}$.

edge positions at half-maximum. As seen in the latter figure, the band narrows significantly in both solvents during the first $50 \mathrm{ps}$. Both edges are shifted toward the center of the band, by about $0.2 \mathrm{~cm}^{-1}$ in ACN, and by about $1-1.5 \mathrm{~cm}^{-1}$ in DCM. The absolute value of this narrowing in the latter solvent is difficult to determine as it depends on the width of the band at early time where the signal-to-noise ratio is rather poor. In DCM, the spectral narrowing is finished after this initial phase, whereas in ACN, a further narrowing, mostly on the blue edge of the band, is observed on a time scale of several hundreds of picoseconds. Although the magnitude of the shift is smaller than the pixel to pixel spectral interval, the observed narrowing is significant, as the analysis is based on the whole band and as the shape of the resulting curves is smooth. Quantitatively, the error of interest is the uncertainty in the difference of fwhm values at corresponding time delays. Assuming that the nar-

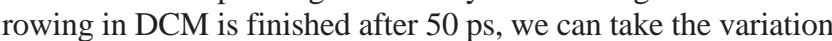
of the fwhm values for longer time delays as the uncertainty of our data evaluation. This yields an error limit of $0.1 \mathrm{~cm}^{-1}$ for the comparison of the two narrowing curves. A further estima- 


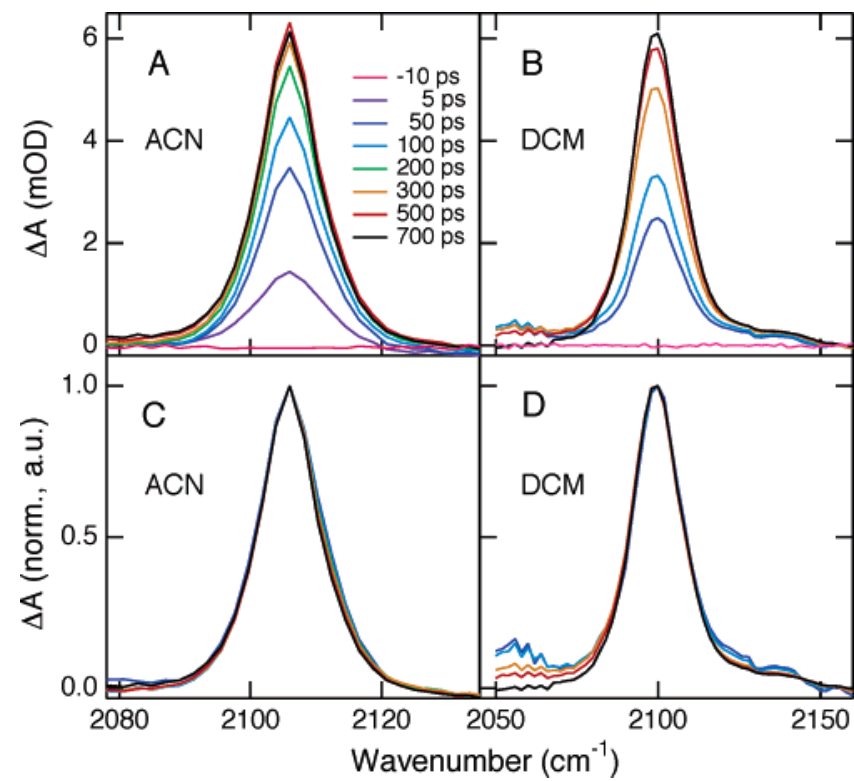

Figure 4. (A), (B) Comparison of the $\mathrm{CN}$ stretching band of $\mathrm{DCB}^{{ }^{-}}$ measured at different time delays after excitation of $\mathrm{Pe}$ and $0.16 \mathrm{M}$ DCB in ACN and DCM. (C), (D) Same as above but after intensity normalization of the spectra measured after 50 ps (the color code is the same for all panels).

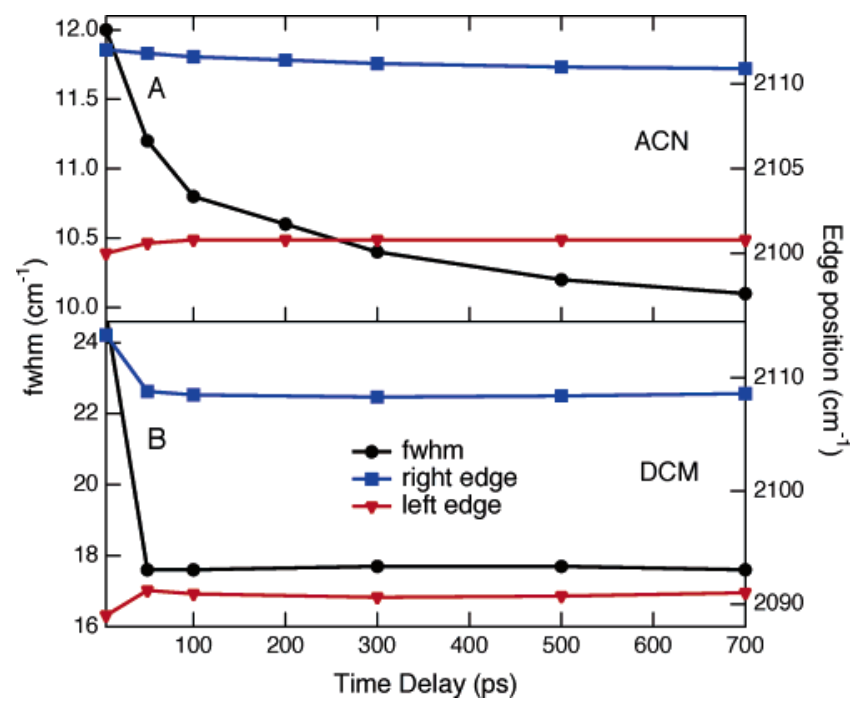

Figure 5. Temporal evolution of the band-shape parameters resulting from the analysis of the $\mathrm{CN}$ stretching band of $\mathrm{DCB}^{--}$in $\mathrm{ACN}$ (A) and DCM (B).

tion of the absolute uncertainty in the fwhm values is provided by the evaluation procedure itself and leads to the same estimated error. When the spectra obtained in ACN are normalized, a small background of about $0.1-0.2 \mathrm{mOD}$ with a slight variation over the spectrum is present on some curves. Subtracting these offsets and re-normalizing in a way that all spectra match at the maximum and either at the red or at the blue end leads to a variation of the difference between fwhm values within $0.1 \mathrm{~cm}^{-1}$.

A monoexponential function cannot be satisfactorily fitted to the temporal evolution of the bandwidth. Although a double exponential fit to a set containing only a few data points does not yield a very significant result, it can be used to estimate the order of magnitude of the time scales involved. The data points can reasonably be reproduced using a pair of time constants with values of $20-40$ and $200-400$ ps. We can thus conclude that the spectral dynamics in ACN is biphasic with the first component to be observed in both ACN and DCM and taking place on a time scale of the order of ten to several tens of picoseconds. This component can be attributed to the widely observed feature of vibrational cooling, ${ }^{15,49,50}$ indicating that a significant amount of excess energy is channeled into vibrational modes of $\mathrm{DCB}^{--}$upon ET. This is somewhat surprising considering the rather small ET driving force, especially in DCM, calculated for this DA pair. Indeed, vibrationally excited states of the ET product are usually supposed to only play a role in highly exergonic reactions. ${ }^{6}$ The second and slower bandnarrowing component is observed in ACN only. The most prominent difference between the two solvents is that in $\mathrm{ACN}$, the geminate ion pairs dissociate into free ions with an efficiency of $30 \%,{ }^{14}$ whereas in DCM, the solvent polarity is not sufficient to compensate for the Coulombic stabilization of the ion pair, and therefore, no separation occurs. Vibrational cooling of ion pairs generated within the first picoseconds after photoexcitation as origin of this second and slower component can be ruled out due to the time scale of several hundreds of picoseconds, which is clearly too long for such a process. Another scenario could be invoked, explaining the remaining broadening by the contribution of "fresh" geminate ion pairs, which are formed at this time delay. As the kinetics depicted in Figure 3B-D show, ET quenching is not complete until some hundreds of picoseconds. Such "fresh" products could still carry the excess energy released upon ET and contribute with a broader band shape. However, taking into account the kinetics of the product formation in DCM shown in Figure 3D, one should expect a second component with an even slower narrowing in this solvent. On the other hand, if the remaining broadening were only due to vibrational cooling of delayed ion formation, the absence of a second component would imply a very prompt ion formation in DCM. This is in clear disagreement with the comparison of the kinetics in Figure 3D, which shows that ET is slower in DCM because of the smaller polarity of this solvent.

In our opinion, the most straightforward interpretation of the observed slow narrowing component in $\mathrm{ACN}$ is the transition from geminate ion pairs into free solvated ions. This hypothesis gets further support by the results of the all-visible pumppump-probe experiment on the same DA pair published recently (see Figure 1). ${ }^{14}$ There, the ground state recovery of $\mathrm{Pe}^{\bullet+}$ generated upon excitation of $\mathrm{Pe}$ at $400 \mathrm{~nm}$ was measured as a function of time delay between the first pump pulse and the subsequent pump-probe experiment on the $\mathrm{D}_{0}-\mathrm{D}_{5}$ electronic transition of $\mathrm{Pe}^{\bullet+}$. At short time delays, the ground state recovery shows a biphasic pattern, but at longer time delays, the dynamics changes to a single-exponential pattern. These findings were interpreted as follows. Just after generation, the ions are close together and $\mathrm{Pe}^{\bullet+}\left(\mathrm{D}_{5}\right)$ may either relax to $\mathrm{Pe}^{\bullet+}$ $\left(\mathrm{D}_{0}\right)$ by internal conversion or undergo charge recombination to the neutral precursor state, $\operatorname{Pe}\left(\mathrm{S}_{1}\right)-\mathrm{DCB}$, followed by a second ET step. A test measurement using trans-1,3-dicyanoethylene as quencher yields a biexponential ground state recovery of $\mathrm{Pe}^{\bullet+}$ at any waiting time after photoexcitation. With this DA pair, the free ion yield is zero as charge recombination is much too fast for separation to be competitive, and therefore, the second reaction channel remains active as long as (paired) ions are present in solution. An analysis of the amplitudes in the corresponding set of rate equations with DCB yields a time constant for the decay of the ion pair population by both separation into free ions and charge recombination of the order of several hundreds of picoseconds, which is, thus, in perfect agreement with the findings of the present investigation. Indeed, the time constant related to the spectral narrowing is not simply 
equal to the time constant of separation, $\tau_{\mathrm{SEP}}$, but corresponds to the decay time of the ion pair population given by $\tau_{\mathrm{IP}}{ }^{-1}=$ $\tau_{\mathrm{SEP}}{ }^{-1}+\tau_{\mathrm{CR}}{ }^{-1}$, where $\tau_{\mathrm{CR}}$ is the time constant of charge recombination. Knowledge of the free ion yield, $\Phi_{\text {ion }}=\tau_{\mathrm{IP}} /$ $\tau_{\mathrm{SEP}}$, which amounts to 0.3 for Pe/DCB in $\mathrm{ACN}$, allows both $\tau_{\mathrm{SEP}}$ and $\tau_{\mathrm{CR}}$ to be estimated (Figure 1 ).

It has to be noted that the mentioned pump-pump-probe attempt is seriously limited to selected DA pairs by technical constrains. The method of a band shape analysis presented here has in contrast the potential for a more general applicability.

The changes of the $\mathrm{CN}$ band shape in $\mathrm{ACN}$ are too small to allow unequivocal structural information on the ion pair to be deduced. Nevertheless, the larger narrowing on the blue side of the band points toward a slight increase of the excess charge density on the cyano-groups upon dissociation into free ions. Weakly exergonic ET quenching reactions have been shown to result in exciplex-like intermediates with an incomplete charge transfer character. ${ }^{51}$ Therefore, the observed changes of band shape might reflect the completion of the charge transfer upon dissociation of the ion pair.

\section{Concluding Remarks}

Using the donor-acceptor pair perylene-1,4-dicyanobenzene as model system, bimolecular electron transfer has been investigated for the first time by means of femtosecond timeresolved mid-IR transient absorption spectroscopy. In addition to confirming what has already been concluded indirectly from all-visible experiments, we are able to extract further information by analyzing the shape of the various observed transient absorption bands as a function of time delay after photoexcitation. The peak narrowing of the antisymmetric $\mathrm{CN}$ stretching vibration of the DCB radical anion on a time scale of a few tens of picoseconds is a direct indication that vibrational excited states of the reaction product are involved even in weakly to moderately exergonic electron transfer processes. The slower narrowing in acetonitrile taking place on a time scale of several hundreds of picoseconds and the comparison with a recently published pump-pump-probe experiment on the same donoracceptor pair, ${ }^{22}$ permits a direct insight into the dissociation of a geminate ion pair into free ions. These results demonstrate the potential of time-resolved mid-IR spectroscopy applied to study bimolecular electron transfer reactions, promising new insight into structural dynamics during the ongoing reaction. Such investigation should yield a clear and direct picture of the different stages of a bimolecular electron transfer reaction.

Acknowledgment. We thank the Laserlab Europe consortium (Project mbi001176) and the Swiss National Science Foundation for the financial support (Project 200020-107466). O.F.M.'s stay at the Max Born Institute was funded through a long-term fellowship of the Egyptian Government.

\section{References and Notes}

(1) Leonhart, H.; Weller, A. Z. Phys. Chem. N. F. 1961, 277.

(2) Weller, A. Pure Appl. Chem. 1982, 54, 1885.

(3) Gould, I. R.; Farid, S. Acc. Chem. Res. 1996, 29, 522.

(4) Mataga, N.; Chosrowjan, H.; Taniguchi, S. J. Photochem. Photobiol. C 2005, 6, 37 .

(5) Vauthey, E. J. Photochem. Photobiol. A 2006, 179, 1.

(6) Marcus, R. A.; Sutin, N. Biochim. Biophys. Acta 1985, 811, 265.

(7) Burshtein, A. I. Adv. Chem. Phys. 2000, 114, 419.
(8) Barzykin, A. V.; Frantsuzov, P. A.; Seki, K.; Tachiya, M. Adv. Chem. Phys. 2002, 123, 511.

(9) Grätzel, M. J. Photochem. Photobiol. C 2003, 4, 145.

(10) Monroe, B. M.; Weed, G. C. Chem. Rev. 1993, 93, 435.

(11) Ottolenghi, M. Acc. Chem. Res. 1973, 6, 153

(12) Takaya, T.; Hamaguchi, H.-o.; Kuroda, H.; Iwata, K. Chem. Phys. Lett. 2004, 399, 210.

(13) Werner, H. J.; Staerk, H.; Weller, A. J. Chem. Phys. 1978, 68, 2419

(14) Pagès, S.; Lang, B.; Vauthey, E. J. Phys. Chem. A 2006, 110, 7547.

(15) Nibbering, E. T. J.; Fidder, H.; Pines, E. Annu. Rev. Phys. Chem. 2005, $56,337$.

(16) Abbott, L. C.; Arnold, C. J.; Ye, T.-Q.; Gordon, K. C.; Perutz, R. N.; Hester, R. E.; Moore, J. N. J. Phys. Chem. A 1998, 102, 1252.

(17) Busby, M.; Matousek, P.; Towrie, M.; Clark, I. P.; Motevalli, M.; Hartl, F.; Vlcek, A., Jr. Inorg. Chem. 2004, 43, 4523.

(18) Kwok, W. M.; Ma, C.; Matousek, P.; Parker, A. W.; Phillips, D.; Toner, W. T.; Towrie, M.; Umapathy, S. J. Phys. Chem. A 2001, 105, 984.

(19) Werncke, W.; Wachsmann-Hogiu, S.; Dreyer, J.; Vodchits, A. I.; Elsaesser, T. Bull. Chem. Soc. Jpn. 2002, 75, 1049.

(20) Hviid, L.; Verhoeven, J. W.; Brouwer, A. M.; Paddon-Row: M. N.; Yang, J. Photochem. Photobiol. Sci. 2004, 3, 246.

(21) Rubtsov, I. V.; Redmore, N. P.; Hochstrasser, R. M.; Therien, M. J. J. Am. Chem. Soc. 2004, 126, 2684.

(22) Maiti, S.; Cowen, B. R.; Diller, R.; Iannone, M.; Moser, C. C.; Dutton, P. L.; Hochstrasser, R. M. Proc. Nat. Acad. Sci. U.S.A. 1993, 90, 5247.

(23) Hamm, P.; Zurek, M.; Mäntele, W.; Meyer, M.; Scheer, H.; Zinth, W. Proc. Nat. Acad. Sci. U.S.A. 1995, 92, 1826.

(24) Heimer, T. A.; Heilweil, E. J. J. Phys. Chem. B 1997, 101, 10990

(25) Asbury, J. B.; Ellingson, R. J.; Ghosh, H. N.; Ferrere, S.; Nozik, A. J.; Lian, T. J. Phys. Chem. B 1999, 103, 3110.

(26) Takeshita, K.; Sasaki, Y.; Kobashi, M.; Tanaka, Y.; Maeda, S ; Yamakata, A.; Ishibashi, T.; Onishi, H. J. Phys. Chem. B 2004, 108, 2963. (27) Wang, C.; Akhremitchev, B.; Walker, G. C. J. Phys. Chem. 1997, 101,2735

(28) Sasaki, Y.; Hamaguchi, H. J. Chem. Phys. 1999, 110, 9179.

(29) Boilet, L.; Buntinx, G.; Lefumeux, C.; Poizat, O. J. Photochem. Photobiol. A 2004, 163, 529 .

(30) Hub, W.; Schneider, S.; Dörr, F.; Simpson, J. T.; Oxman, J. D.; Lewis, F. D. J. Am. Chem. Soc. 1982, 104, 2044.

(31) Fournier, T.; Tavender, S. M.; Parker, A. W.; Scholes, G. D.; Phillips, D. J. Phys. Chem. A 1997, 101, 5320.

(32) Poizat, O.; Buntinx, G.; Boilet, L. J. Phys. Chem. A 2005, 109, 10813

(33) Vauthey, E.; Phillips, D.; Parker, A. W. J. Phys. Chem. 1992, 96, 7356.

(34) Vauthey, E.; Parker, A. W.; Phillips, D.; Nohova, B. J. Am. Chem Soc. 1994, 116, 9182

(35) Vauthey, E. J. Phys. Chem. A 2000, 104, 1804

(36) Vauthey, E. J. Phys. Chem. A 2001, 105, 340.

(37) Matousek, P.; Towrie, M.; Parker, A. W. J. Raman Spectrosc. 2002, 33,238

(38) Rini, M.; Kummrow, A.; Dreyer, J.; Nibbering, E. T. J.; Elsaesser, T. Faraday Discuss. 2003, 122, 27.

(39) Kaindl, R. A.; Wurm, M.; Reimann, K.; Hamm, P.; Weiner, A. M.; Woerner, M. J. Opt. Soc. Am. B 2000, 17, 2086.

(40) Greever, J. S.; Turner, J. B. M.; Kauffman, H. F. J. Phys. Chem A 2003, 107, 4072 .

(41) Vauthey, E.; Suppan, P.; Haselbach, E.; Davidson, R. S. Helv. Chim. Acta. 1986, 69, 430.

(42) Szczepanski, J.; Chapo, C.; Vala, M. Chem. Phys. Lett. 1993, 205, 434.

(43) Chudoba, C.; Nibbering, E. T. J.; Elsaesser, T. Phys. Rev. Lett. 1998, 81,3010

(44) Nibbering, E. T. J.; Chudoba, C.; Elsaesser, T. Isr. J. Chem. 1999, 39,333

(45) Palit, D. K.; Zhang, T. Q.; Kumazaki, S.; Yoshihara, K. J. Phys. Chem. A 2003, 107, 10798 .

(46) Asbury, J. B.; Wang, Y. Q.; Lian, T. Q. Bull. Chem. Soc. Jpn. 2002, 75, 973

(47) Shida, T. Electronic Absorption Spectra of Radical Ions; Elsevier: Amsterdam, 1988; Vol. Physical Sciences data 34.

(48) Weller, A. Z. Phys. Chem. N. F. 1982, 133, 93

(49) Elsaesser, T.; Kaiser, W. Аnnu. Rev. Phys. Chem. 1991, 42, 83.

(50) Owrutsky, J. C.; Raftery, D.; Hochstrasser, R. M. Annu. Rev. Phys. Chem. 1994, 45, 519 .

(51) Kuzmin, M. G. J. Photochem. Photobiol. A 1996, 102, 51. 